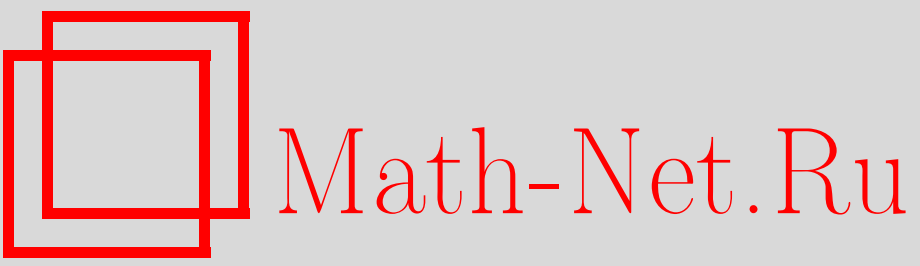

А. А. Джалилов, Кусочная гладкость сопряжений гомеоморфизмов окружности с особенностями типа излома, ТМФ, 1999, том 120, номер 2, 179-192

DOI: https://doi.org/10.4213/tmf768

Использование Общероссийского математического портала Math-Net.Ru подразумевает, что вы прочитали и согласны с пользовательским соглашением

http://www.mathnet.ru/rus/agreement

Параметры загрузки:

IP: 18.234 .156 .22

26 апреля 2023 г., $17: 12: 07$ 


\section{КУСОЧНАЯ ГЛАДКОСТЬ СОПРЯЖЕНИЙ ГОМЕОМОРФИЗМОВ ОКРУЖНОСТИ С ОСОБЕННОСТЯМИ ТИПА ИЗЛОМА}

Доказано, что сопряжение для некоторых гомеоморфизмов окружности с изломами, лежащими на одной траектории, является кусочно-гладкой функцией.

\section{1. ВВЕДЕНИЕ}

В настоящей работе изучаются гомеоморфизмы окружности с особенностями типа излома, т.е. отображения гладкие всюду, за исключением нескольких точек, в которых происходит разрыв первой производной. Рассмотрим сохраняющий ориентацию гомеоморфизм $T_{f} x$ единичной окружности

$$
T_{f} x=\{f(x)\}, \quad x \in S^{1}=[0,1),
$$

где скобка $\{\cdot\}$ обозначает дробную часть числа, а $f(x)$ - поднятие, определяюшее $T_{f}$, которое удовлетворяет следующим условиям:

а) $f(x)$ - непрерывная, строго возрастаюшая функция;

б) $f(x+1)=f(x)+1$ для любого $x \in R^{1}$;

в) гомеоморфизм $T_{f}$ в точках $x_{p_{i}}=T_{f}^{p_{i}} x_{0}, i=\overline{0, m}, p_{0}=0<p_{1}<p_{2}<\cdots<p_{m}$, имеет изломы; кроме того, сушествуют конечные одностронние производные $f^{\prime}\left(x_{p_{i}} \pm 0\right)$ и

$$
\sqrt{\frac{f^{\prime}\left(x_{p_{i}}-0\right)}{f^{\prime}\left(x_{p_{i}}+0\right)}}=c_{i}(f) \neq 1, \quad i=\overline{0, m}
$$

г) $f(x) \in C^{2+\varepsilon}\left(S^{1} \backslash\left\{x_{p_{i}}, i=\overline{0, m}\right\}\right)$ при некотором $\varepsilon>0, f^{\prime}(x) \geqslant$ const $>0, x \in$ $S^{1} \backslash\left\{x_{p_{i}}, i=\overline{1, m}\right\}$;

д) $\prod_{i=0}^{m} c_{i}=1$.

* Самаркандский государственный университет, Самарканд, Узбекистан. E-mail:info@mchmat.samuni.silk.org 
ЗАмЕчАниЕ. Условие "г" означает, что функция $f(x)$ принадлежит классу $C^{2+\varepsilon}$ на каждой связной компоненте множества $S^{1} \backslash\left\{x_{p_{i}}, i=\overline{0, m}\right\}$.

Важной характеристикой гомеоморфизмов окружности является число врашения (см. [1])

$$
\rho(f)=\lim _{n \rightarrow \infty} \frac{f^{n}(x)}{n} \quad(\bmod 1) .
$$

Здесь и далее $f^{(n)}$ обозначает $n$-ю итерацию функции (или отображения $f$ ). Хорошо известно, что предел существует и не зависит от начальной точки $x \in R^{1}$.

Условия "а"- “д” означают, что функция $f(x)$ определяет гомеоморфизм окружности, достаточно гладкий всюду, за исключением $(m+1)$ точек окружности, в которых имеются скачки первой производной или изломы. Условие “д” в определенном смысле компенсирует наличие точек излома у функции $f(x)$.

Пусть число вращения $\rho(f)$ иррационально. Данжуа показал [2], что если $f \in C^{1}\left(R^{1}\right)$ и $\operatorname{var}_{S^{1}} \ln f^{\prime}(x)<\infty$, то сушествует гомеоморфизм окружности $T_{\varphi}$ такой, что

$$
T_{\varphi} \circ T_{f}=T_{\rho} \circ T_{\varphi}
$$

где $T_{\rho} x=\{x+\rho\}$ - линейный поворот окружности на угол $\rho$. В теории гомеоморфизмов окружности сушествует важная проблема выяснения связи между гладкостью $f$, свойствами числа врашения $\rho$ и классом гладкости сопряжения $\varphi[3,4]$. С этой проблемой тесно связан и вопрос о сушествовании абсолютно непрерывной инвариантной меры для $T_{f}$. В самом деле, единственная с точностью до нормировки инвариантная мера для $T_{f}$ является абсолютно непрерьвной относительно меры Лебега тогда и только тогда, когда $\varphi(x)$ - абсолютно непрерывная функция. Это соображение впервые было использовано Арнольдом в [3], где он изучал гладкость $\varphi(x)$. Там же было показано, что для типичных чисел врашения $\rho$ и для аналитических диффеоморфизмов $T_{f}$, достаточно близких к повороту $T_{\rho}$, приведение осушествляется с помощью аналитического диффеоморфизма. Результаты типа теоремы Арнольда, получили название локальных теорем приведения (ЛТП). Позже Мозер доказал ЛТП для достаточно гладких, но не аналитических, диффеоморфизмов [5].

Глобальная теорема о гладкости приведения была решена Эрманом [4]. Наиболее сильные результаты в этой области были получены Катцнельсоном, Орнштейном [6], Ханиным и Синаем $[7,8]$ и Старком [9]. Работы [6-10] можно отнести к ренормгрупповому подходу в теории динамических систем. Достоинством подхода работы Синая и Ханина [8] является то, что в ней использовано приближение итераций изучаемых гомеоморфизмов с помощью дробно-линейных отображений.

Гомеоморфизмы окружности с одной точкой излома были изучены в работах [10-12]. В работах $[10,11]$ показано, что гомеоморфизмы с одной точкой излома занимают промежуточное место между диффеоморфизмами, отвечающими $c=1$, и критическими отображениями окружности. С одной стороны, для однопараметрических семейств гомеоморфизмов с особенностями типа излома множество значений параметра, отвечающих 
иррациональным числам врашения, имеет лебегову меру нуль, а сама динамика характеризуется нетривиальными масштабными преобразованиями, или скейлингами. Инвариантные меры таких гомеоморфизмов являются сингулярными относительно меры Лебега [12].

С другой стороны, как и в случае диффеоморфизмов окружности, ренормгрупповое поведение для подобных отображений выглядит достаточно просто. В перенормированных координатах итерации отображений асимтотически приближаются к дробно-линейным преобразованиям, зависящим только от двух параметров.

Основной целью настоящей работы является доказательство следующего результата.

Теорема 1.1. Предположим, что поднятие $f(x)$ гомеоморфизма $T_{f}$ удовлетворяет условиям "а"-“д". Число вращения $\rho(f)$ иррационально, и его разложсение в непрерывную дробь имеет вид $\rho(f)=\left[k_{1}, k_{2}, \ldots, k_{n}, \ldots\right]$. Тогда:

1) $п р и k_{n} \leqslant$ const $\partial$ яя всех $n \geqslant 1$

$$
T_{\varphi} \in C^{1+\varepsilon}\left(S^{1} \backslash\left\{x_{i}, 1 \leqslant i \leqslant p_{m}\right\}\right)
$$

2) $п р и k_{n} \leqslant$ const $n^{\alpha}$ для некоторого значения $\alpha$

$$
T_{\varphi} \in C^{1+\nu}\left(S^{1} \backslash\left\{x_{i}, 1 \leqslant i \leqslant p_{m}\right\}\right)
$$

для $л ю б о г о ~ \nu<\varepsilon ;$

3) в предположсениях 1 и 2 сопряжение $T_{\varphi}$ в точках $x=x_{i}, \quad 1 \leqslant i \leqslant p_{m}$, имеет изломиц

$$
\frac{\varphi^{\prime}\left(x_{i}-0\right)}{\varphi^{\prime}\left(x_{i}+0\right)}=c_{j} c_{j+1} \cdots c_{m}
$$

npu $p_{j-1}<i \leqslant p_{j}, \quad j=\overline{1, m}$.

Работа имеет следующую структуру. В разделе 2 приводятся необходимые определения и факты. В разделе 3 изучается ренормгрупповое поведение и доказывается сходимость к линейным отображениям. В разделе 4 содержится доказательство теоремы 1.1.

\section{2. НЕОБХОДИМЫЕ ОПРЕДЕЛЕНИЯ И ФАКТЫ}

Рассмотрим сохраняющий ориентацию гомеоморфизм окружности $T_{f}$, определяемый поднятием $f(x), x \in R^{1}$, т.е. $T_{f} x=\{f(x)\}, x \in S^{1}$.

Всюду в дальнейшем будем предполагать число врашения $\rho(f)$ иррациональным. Возьмем произвольную точку $x_{0} \in S^{1}$ и рассмотрим траекторию этой точки $\left\{x_{i}=T^{i} x_{0}, i \in Z^{1}\right\}$. Согласно классической теореме Пуанкаре (см. [1]) порядок точек вдоль этой траектории будет в точности таким же, как и для линейного поворота окружности $T_{\rho}$, т.е. для последовательности $\left\{\bar{x}_{i}=\left\{x_{0}+i \rho\right\}, i \in Z^{1}\right\}$. Это важное свойство позволяет определить систему естественных разбиений окружности, 
связанных с разложением $\rho(f)$ в непрерывную дробь. Пусть разложение $\rho(f)$ в непрерывную дробь имеет вид $\rho(f)=\left[k_{1}, k_{2}, \ldots, k_{n}, \ldots\right]$. Обозначим

$$
\frac{p_{n}}{q_{n}}=\left[k_{1}, k_{2}, \ldots, k_{n}\right], \quad n \geqslant 1
$$

Числа $p_{n}$ и $q_{n}$ удовлетворяет следуюшим рекуррентным соотношениям:

$$
\begin{aligned}
p_{n+1}=k_{n+1} p_{n}+p_{n-1}, & p_{0}=0, & p_{1}=1, \\
q_{n+1}=k_{n+1} q_{n}+q_{n-1}, & q_{0}=0, & q_{1}=k_{1} .
\end{aligned}
$$

Пусть $x_{0} \in S^{1}$. Обозначим через $\triangle_{0}^{(n)}\left(x_{0}\right)$ замкнутый интервал с концами в точках $x_{0}$ и $x_{q_{n}}=T^{q_{n}} x_{0}$. Заметим, что при нечетном $n$ точка $x_{q_{n}}$ лежит слева от $x_{0}$, а при четном $n$ - справа. Обозначим $\Delta_{i}^{(n)}\left(x_{0}\right)=T^{i} \Delta_{0}^{(n)}\left(x_{0}\right), i \geqslant 1$.

Tеорема 2.1 (см. [13]). Рассмотрим произвольную точку $x_{0} \in S^{1}$. Отрезок траектории этой точки $\left\{x_{i}, 0 \leqslant i<q_{n}+q_{n-1}\right\}$ разбивает окружность на непересекающиеся (за исключением концевых точек) отрезки

$$
\Delta_{k}^{(n-1)}\left(x_{0}\right), \quad 0 \leqslant k<q_{n}, \quad \Delta_{j}^{(n)}\left(x_{0}\right), \quad 0 \leqslant j<q_{n-1} .
$$

Полученное разбиение обозначим через $\xi_{n}\left(x_{0}\right)$ и назовем динамическим разбиением $n$-го порядка. Опишем теперь процесс перехода от разбиения $\xi_{n}\left(x_{0}\right)$ к $\xi_{n+1}\left(x_{0}\right)$. Все отрезки $\Delta_{j}^{(n)}\left(x_{0}\right), 0 \leqslant j \leqslant q_{n-1}-1$, сохраняются, а каждый из отрезков $\Delta_{i}^{(n-1)}\left(x_{0}\right)$, $0 \leqslant i \leqslant q_{n}-1$, разбивается на $\left(k_{n+1}+1\right)$ отрезков:

$$
\Delta_{i}^{(n-1)}\left(x_{0}\right)=\Delta_{i}^{(n+1)}\left(x_{0}\right) \bigcup_{s=0}^{k_{n+1}-1} \Delta_{i+q_{n-1}+s q_{n}}^{(n)}\left(x_{0}\right) .
$$

Очевидно, что $\xi_{1}\left(x_{0}\right) \leqslant \xi_{2}\left(x_{0}\right) \leqslant \cdots \leqslant \xi_{n}\left(x_{0}\right) \leqslant \cdots$.

ЛЕмма ДАнжУА. Пусть в точках $z^{(i)} \in S^{1}, \quad i=\overline{0, r}, \quad z^{(1)}<z^{(2)}<\cdots<z^{(r)}$, существуют конечние односторонние производние $f^{\prime}\left(z^{(i)} \pm 0\right)>0, \quad f \in$ $C^{1}\left(\left[z^{(i)}, z^{(i+1)}\right]\right), \quad i=\overline{0, r}, \quad z^{(r+1)}=z^{(1)}+1, u$

$$
\sum_{i=1}^{r} \operatorname{var}_{\left[z^{(i)}, z^{(i+1)}\right]} \ln f^{\prime}=\bar{v}<\infty
$$

Положим

$$
v=\bar{v}+\sum_{i=0}^{r}\left|\ln f^{\prime}\left(z^{(i)}-0\right)-\ln f^{\prime}\left(z^{(i)}+0\right)\right| .
$$

Тогда для любого $y_{0}$ такого, что $y_{s} \notin\left\{z^{(i)}, i=\overline{1, r}\right\}, \quad s=\overline{0, q_{k}-1}, \quad k \in N$, справедливо неравенство

$$
e^{-v} \leqslant \prod_{j=0}^{q_{k}-1} f^{\prime}\left(y_{j}\right) \leqslant e^{v}
$$


ЛЕмма 2.1. Пусть выполнены условия леммы Данжуа. Тогда для любого $y_{0} \in S^{1}$ выполнено неравенство

$$
\left|\Delta_{0}^{(n)}\left(y_{0}\right)\right| \leqslant \lambda^{2 k}\left|\Delta_{0}^{(n-2 k)}\left(y_{0}\right)\right|, \quad 0 \leqslant 2 k \leqslant n,
$$

где $\lambda=\left(1+e^{-v}\right)^{-1 / 2}<1, a|\cdot|$ обозначает длину отрезка.

Леммы Данжуа и 2.1 доказываются так же, как и аналогичные утверждения в гладком случае (см. [8]).

Из леммы 2.1 следует, что отрезки, составляюшие динамическое разбиение $\xi_{n}\left(y_{0}\right)$, имеют экспоненциально малую длину.

СлеДСТВИЕ. Пусть $\Delta^{(n)}$ - произвольный элемент динамического разбиения $\xi_{n}\left(y_{0}\right)$, тогда для всех $n \geqslant 1$ справедливо неравенство

$$
\left|\Delta^{(n)}\right| \leqslant \operatorname{const} \lambda^{n}
$$

Из формулы (2.2) следует, что траектория любой точки всюду плотна на окружности.

ТЕОРЕМА ДАНжУА. Пусть въполнены условия леммы Данжуа. Тогда гомеоморфизм $T_{f}$ топологически сопряжен линейному повороту $T_{\rho}$.

ДокАЗАТЕЛЬСТво. Возьмем две произвольные точки $y_{0}$ и $z_{0}$ и рассмотрим их траектории под действием соответствующих гомеоморфизмов $T_{f}$ и $T_{\rho}$, т.е. $\left\{y_{i}=T_{f}^{i} y_{0}, i \geqslant 0\right\}$ и $\left\{z_{i}=T_{\rho}^{i} z_{0}, i \geqslant 0\right\}$. Определим сопряжение $T_{\varphi}$ на множестве $\left\{y_{i}, i \geqslant 0\right\}$ следующим образом: $T_{\varphi} y_{i}=z_{i}, i \geqslant 0$. Отображение $T_{\varphi}$ удовлетворяет (1.2), и, поскольку траектория точки $x_{0}$ всюду плотна, его можно продолжить по непрерывности на всю окружность.

Теорема Данжуа доказана.

Из доказательства теоремы Данжуа видно, что если траектория некоторой точки под действием $T_{f}$ плотно заполняет окружность, то сопряжение $T_{\varphi}$ определяется единственным образом с точностью до поворота. Кроме того, поскольку число врашений $\rho(f)$ иррационально, $T_{f}$ является строго эргодическим [1], т.е. обладает единственной нормированной инвариантной мерой $\mu$. Хорошо известно, что

$$
T_{\varphi} x=\int_{x_{0}}^{x} d \mu(y), \quad x \in S^{1} .
$$

Отсюда следует, что если инвариантная мера абсолютно непрерывна, то ее плотность $h(x)$ удовлетворяет следуюшему гомологическому уравнению:

$$
h\left(T_{f} x\right)=\frac{1}{f^{\prime}(x)} h(x) .
$$

Таким образом, вопрос о гладкости сопряжения сводится к вопросу о построении плотности инвариантной меры и исследовании ее гладкости. 


\section{3. РЕНОРМГРУППОВОЕ ПОВЕДЕНИЕ ГОМЕОМОРФИЗМА $T_{f}$}

В этом разделе мы изучаем ренормгрупповое поведение гомеоморфизма $T_{f}$ в окрестности особой точки $x=x_{0}$. Обозначим через $n_{0}$ минимальное четное число, удовлетворяющее условию $q_{n_{0}-1}>p_{m}$.

Рассмотрим разбиение $\xi_{n_{0}}\left(x_{0}\right)$ и окрестность $V_{0}=\left[x_{q_{n_{0}-1}}, x_{q_{n_{0}}}\right]$ точки $x_{0}$. Из структуры разбиения $\xi_{n_{0}}\left(x_{0}\right)$ и определения $n_{0}$ следует, что отрезок $V_{0}$ не содержит других особых точек, кроме $x=x_{0}$, и все остальные особые точки лежат только в первых $p_{m}$ образах отрезка $V_{0}$. Обозначим через $l_{0}$ минимальное число такое, что отрезки $\Delta_{0}^{\left(l_{0}-1\right)}\left(x_{k}\right)$ и $\Delta_{0}^{\left(l_{0}\right)}\left(x_{k}\right), k=q_{n_{0}-1}, q_{n_{0}}$, и их первые $p_{m}$ образов не содержат особых точек. Обозначим через $A$ левую крайнюю точку отрезка $\Delta_{0}^{\left(l_{0}-1\right)}\left(x_{q_{n_{0}-1}}\right)$ и через $B$ правую крайнюю точку отрезка $\Delta_{0}^{\left(l_{0}\right)}\left(x_{q_{n_{0}}}\right)$. Ясно, что $V_{0} \subset[A, B]$. Положим $l=\max \left\{n_{0}, l_{0}\right\}$.

Возьмем произвольную точку $y_{0} \in\left[x_{q_{n_{0}-1}}, x_{q_{n_{0}}}\right]$ и рассмотрим динамическое разбиение $\xi_{n}\left(y_{0}\right)$. Положим для определенности $n$ четным. Тогда

$$
\begin{aligned}
\Delta_{i}^{(n-1)}\left(y_{0}\right) & =\left[y_{q_{n-1}+i}, y_{i}\right], & & 0 \leqslant i \leqslant q_{n}-1, \\
\Delta_{j}^{(n)}\left(y_{0}\right) & =\left[y_{i}, y_{q_{n}+i}\right], & & 0 \leqslant j \leqslant q_{n-1}-1 .
\end{aligned}
$$

Следуя методу ренормгруппы, мы изучаем при $n \rightarrow \infty$ асимптотические поведения отображений

$$
\begin{array}{r}
T^{q_{n}}:\left[y_{q_{n-1}}, y_{0}\right] \longrightarrow\left[y_{q_{n-1}+q_{n}}, y_{q_{n}}\right] \\
T^{q_{n-1}}:\left[y_{0}, y_{q_{n}}\right] \longrightarrow\left[y_{q_{n-1}}, y_{q_{n-1}+q_{n}}\right],
\end{array}
$$

используя в окрестности точки $y_{0}$ перенормированную переменную

$$
z=\frac{y-y_{0}}{y_{0}-y_{q_{n-1}}} .
$$

В координатах $z$ получим, что $y_{0} \rightarrow 0$ и $y_{q_{n-1}} \rightarrow-1$. Обозначим через $a_{n}$ перенормированную координату точки $y_{q_{n}}$ :

$$
a_{n}=\frac{y_{q_{n}}-y_{0}}{y_{0}-y_{q_{n-1}}} .
$$

Используя $\Delta_{q_{n}}^{(n)} \subset \Delta_{0}^{(n-1)}$ и лемму Данжуа, легко получаем, что $0<a_{n}<$ const. В новых координатах отрезкам $\left[y_{q_{n-1}}, y_{0}\right]$ и $\left[y_{0}, y_{q_{n}}\right]$ соответствуют отрезки $[-1,0]$ и $\left[0, a_{n}\right]$. Отображениям $T^{q_{n}}$ и $T^{q_{n-1}}$ в перенормированных координатах отвечают следующие функции:

$$
\begin{array}{ll}
f_{n}(z)=\frac{T^{q_{n}}\left(y_{0}+z\left(y_{0}-y_{q_{n-1}}\right)\right)-y_{0}}{y_{0}-y_{q_{n-1}}}, & z \in[-1,0], \\
g_{n}(z)=\frac{T^{q_{n-1}}\left(y_{0}+z\left(y_{0}-y_{q_{n-1}}\right)\right)-y_{0}}{y_{0}-y_{q_{n-1}}}, & z \in\left[0, a_{n}\right] .
\end{array}
$$

Мы покажем, что функции $f_{n}(z)$ и $g_{n}(z)$ при $n \rightarrow \infty$ асимптотически близки к линейным функциям. Обозначим

$$
F_{n}(z)=z+a_{n}, \quad G_{n}(z)=z-1 .
$$


Teорема 3.1. Пусть $y_{0} \in V_{0}$. Существует константа $C_{1}>0$ такая, что при все $x \geqslant l$

$$
\begin{aligned}
& \left\|f_{n}(z)-F_{n}(z)\right\|_{C^{1}([-1,0])} \leqslant C_{1} \lambda^{n \varepsilon} \\
& \left\|g_{n}(z)-G_{n}(z)\right\|_{C^{1}\left(\left[0, a_{n}\right]\right)} \leqslant C_{1} \lambda^{n \varepsilon} .
\end{aligned}
$$

Введем относительные координаты $z_{i}, 0 \leqslant i \leqslant q_{n}$, на отрезках $\Delta_{i}^{(n-1)}\left(y_{0}\right)$ и $\bar{z}_{j}, 0 \leqslant$ $j \leqslant q_{n-1}$, на отрезках $\Delta_{j}^{(n)}\left(y_{0}\right)$ в соответствии с формулами

$$
z_{i}=\frac{y_{i}-y}{y_{i}-y_{q_{n-1}+i}}, \quad \bar{z}_{j}=\frac{y_{q_{n}+j}-y}{y_{q_{n}+j}-y_{j}} .
$$

Обозначим

$$
\begin{array}{ll}
M(i)=\exp \left\{\sum_{s=0}^{i-1} \int_{x_{s+q_{n-1}}}^{x_{s}} \frac{f^{\prime \prime}(t)}{2 f^{\prime}(t)} d t\right\}, & 0 \leqslant i \leqslant q_{n}, \\
\bar{M}(j)=\exp \left\{\sum_{s=0}^{j-1} \int_{x_{s}}^{x_{s+q_{n}}} \frac{f^{\prime \prime}(t)}{2 f^{\prime}(t)} d t\right\}, & 0 \leqslant i \leqslant q_{n-1}, \\
l_{n-1}=\max _{0 \leqslant k \leqslant q_{n}}\left|\Delta_{k}^{(n-1)}\left(y_{0}\right)\right|, & n \geqslant 2 .
\end{array}
$$

Для простоты записи в дальнейшем через $\|f\|_{1}$ обозначим $C^{1}$-норму функции $f(z)$ на отрезке $[0,1]$.

Чтобы доказать теорему 3.1 , нам понадобятся леммы 3.1 и 3.2 .

Лемма 3.1 (см. [11]):

1) если каждый из отрезков $\Delta_{0}^{(n-1)}\left(y_{0}\right), \Delta_{1}^{(n-1)}\left(y_{0}\right), \ldots, \Delta_{i-1}^{(n-1)}\left(y_{0}\right), \quad 1 \leqslant i \leqslant q_{n}$, не содержит особых точек $x_{p_{s}}, 0 \leqslant s \leqslant m$, то

$$
z_{i}=\frac{M(i) z_{0}}{1+(M(i)-1) z_{0}}+\eta_{i}\left(z_{0}\right)
$$

әде $\left\|\eta_{i}\left(z_{0}\right)\right\|_{1} \leqslant$ const $l_{n-1}^{\varepsilon}$

2) если каждий из отрезков $\Delta_{0}^{(n)}\left(y_{0}\right), \Delta_{1}^{(n)}\left(y_{0}\right), \ldots, \Delta_{j-1}^{(n)}\left(y_{0}\right), \quad 1 \leqslant j \leqslant q_{n-1}$, не содержит особих точек $x_{p_{s}}, 0 \leqslant s \leqslant m$, mо

$$
\bar{z}_{j}=\frac{\bar{M}(j) \bar{z}_{0}}{1+(\bar{M}(j)-1) \bar{z}_{0}}+\bar{\eta}_{j}\left(\bar{z}_{0}\right),
$$

əəe $\left\|\bar{\eta}_{j}\left(\bar{z}_{0}\right)\right\|_{1} \leqslant$ const $l_{n}^{\varepsilon}$.

Отметим, что в формулировке леммы 3.1 и ниже const обозначает константы, зависяшие только от исходного гомеоморфизма и не зависяшие от $n, i, j$ и т.д. 
Смысл леммы 3.1 состоит в том, что $z_{i}$ и $\bar{z}_{j}$ с высокой степенью точности аппроксимируются дробно-линейными функциями от $z_{0}$ и $\bar{z}_{0}$.

Пусть $y_{0} \in V_{0}$. Фиксируем $n \geqslant l$. Из определения $l$ следует, что либо

$$
\left\{x_{i}, 0 \leqslant i \leqslant p_{m}\right\} \subset \bigcup_{s=0}^{q_{n}-1} \Delta_{s}^{(n-1)}\left(y_{0}\right),
$$

либо

$$
\left\{x_{i}, 0 \leqslant i \leqslant p_{m}\right\} \subset \bigcup_{s=0}^{q_{n-1}-1} \Delta_{s}^{(n)}\left(y_{0}\right) .
$$

Пусть для определенности имеет место первый случай и $x_{p_{i}} \in \Delta_{t+p_{i}}^{(n-1)}\left(y_{0}\right), \quad 0 \leqslant t \leqslant$ $q_{n}-p_{m}-1, i=\overline{0, m}, p_{0}=0$.

Теперь мы найдем выражение для $z_{q_{n}}\left(z_{0}\right)$. Положим $\alpha_{n m}=q_{n}-t-p_{m}-1$. Пусть функции $z_{t}(z), z_{p_{m}+1}(z), z_{\alpha_{n m}}(z)$ суть относительные координаты, соответствуюшие отображениям

$$
\begin{aligned}
T^{t}: \Delta_{0}^{(n-1)}\left(y_{0}\right) & \longrightarrow \Delta_{t}^{(n-1)}\left(y_{0}\right), \\
T^{p_{m}+1}: \Delta_{t}^{(n-1)}\left(y_{0}\right) & \longrightarrow \Delta_{t+p_{m}+1}^{(n-1)}\left(y_{0}\right), \\
T^{\alpha_{n m}}: \Delta_{t+p_{m}+1}^{(n-1)}\left(y_{0}\right) & \longrightarrow \Delta_{q_{n}}^{(n-1)}\left(y_{0}\right) .
\end{aligned}
$$

Тогда функцию $z_{q_{n}}\left(z_{0}\right)$ можно представить в виде

$$
z_{q_{n}}\left(z_{0}\right)=z_{\alpha_{n m}}\left(z_{p_{m}+1}\left(z_{t}\left(z_{0}\right)\right)\right)
$$

В случае $t=0$ мы будем рассматривать два последних отображения.

Поскольку цепочки отрезков $\Delta_{i}^{(n-1)}\left(y_{0}\right), \quad i=\overline{0,(t-1)}$, и $\Delta_{k}^{(n-1)}\left(y_{0}\right), \quad k=$ $\overline{\left(t+p_{m}+1\right), q_{n}}$, не содержат особых точек, используя лемму 3.1 , получаем

$$
\begin{aligned}
z_{t}\left(z_{0}\right) & =\frac{N_{1} z_{0}}{1+\left(N_{1}-1\right) z_{0}}+\chi_{1}\left(z_{0}\right), \\
z_{\alpha_{n m}}(z) & =\frac{N_{2} z}{1+\left(N_{2}-1\right) z}+\chi_{2}(z),
\end{aligned}
$$

где

$$
\begin{gathered}
N_{1}=\exp \left\{\sum_{k=0}^{t-1} \int_{y_{k+q_{n-1}}}^{y_{k}} \frac{f^{\prime \prime}(t)}{2 f^{\prime}(t)} d t\right\}, \\
N_{2}=\exp \left\{\sum_{k=t+p_{m}+1}^{q_{n}-1} \int_{y_{k+q_{n-1}}}^{y_{k}} \frac{f^{\prime \prime}(t)}{2 f^{\prime}(t)} d t\right\}, \\
\left\|\chi_{1}(z)\right\|_{1} \leqslant \operatorname{const} l_{n-1}^{\varepsilon}, \quad\left\|\chi_{2}(z)\right\|_{1} \leqslant \operatorname{const} l_{n-1}^{\varepsilon} .
\end{gathered}
$$

Теперь мы покажем, что функция $z_{p_{m}+1}(z)$ является почти тождественной. 
ЛЕмма 3.2. Имеем

$$
z_{p_{m}+1}(z)=z+\chi_{3}(z)
$$

әде $\left\|\chi_{3}(z)\right\|_{1} \leqslant$ const $l_{n-1}^{\varepsilon}$.

ДоказАтЕльство леммы 3.2. Отображение $T$ на отрезках $\Delta_{t+p_{i}}^{(n-1)}\left(y_{0}\right), i=\overline{0, m}$, имеет точки излома. Учитывая это, функцию $z_{p_{m}+1}(z)$ представим в виде

$$
z_{p_{m}+1}(z)=z_{1}^{(m)}\left(z_{p(m)}\left(z_{1}^{(m-1)}\left(z_{p(m-1)} \ldots z_{p(1)}\left(z_{1}^{(0)}(z)\right) \ldots\right)\right)\right)
$$

здесь $p(i)=p_{i}-p_{i-1}-1, i=\overline{1, m}$, а $z_{1}^{(i)}(z), i=\overline{0, m}$, суть относительные координаты, соответствующие отображениям

$$
T: \Delta_{t+p_{i}}^{(n-1)}\left(y_{0}\right) \rightarrow \Delta_{t+p_{i}+1}^{(n-1)}\left(y_{0}\right)
$$

в то время как функции $z_{p(i)}(z)$ являются относительными координатами отображений

$$
T^{p(i)}: \Delta_{t+p_{i-1}+1}^{(n-1)}\left(y_{0}\right) \rightarrow \Delta_{t+p_{i}}^{(n-1)}\left(y_{0}\right), \quad i=\overline{1, m} .
$$

В силу леммы 3.1 для всех $1 \leqslant i \leqslant m$ имеем

$$
z_{p(i)}(z)=\frac{H_{i} z}{1+\left(H_{i}-1\right) z}+\theta_{i}(z)
$$

где $\left\|\theta_{i}(z)\right\|_{1} \leqslant \operatorname{const} l_{n-1}^{\varepsilon}$ и

$$
H_{i}=\exp \left\{\sum_{s=1}^{p(i)} \int_{\Delta_{t+p_{i-1}+s}^{(n-1)}} \frac{f^{\prime \prime}(t)}{2 f^{\prime}(t)} d t\right\} .
$$

Используя оценки $p(i) \leqslant p_{m}, i=\overline{0, q_{n}}$, и $\left|\Delta_{i}^{(n-1)}\left(y_{0}\right)\right| \leqslant$ const $l_{n-1}$, получаем

$$
\left|\ln H_{i}\right| \leqslant \text { const } l_{n-1} \text {. }
$$

Отсюда, а также из (3.8), следует, что

$$
z_{p(i)}(z)=z+h_{i}(z), \quad i=\overline{1, m},
$$

где $\left\|h_{i}(z)\right\|_{1} \leqslant$ const $l_{n-1}^{\varepsilon}$.

Перейдем теперь к функциям $z_{1}^{(i)}(z), i=\overline{0, m}$. Обозначим через $d_{i}, 0 \leqslant i \leqslant m$, относительные координаты особых точек $x=x_{p_{i}}$, т.е.

$$
d_{i}=\frac{y_{t+p_{i}}-x_{p_{i}}}{y_{t+p_{i}}-y_{t+p_{i}+q_{n-1}}} .
$$

Используя разложение Тейлора и условия “в" и “г” из введения, можно легко показать, что

$$
z_{1}^{(i)}(z)=\varphi_{i}(z)+r_{i}(z), \quad 1 \leqslant i \leqslant m
$$

где $\left\|r_{i}(z)\right\|_{1} \leqslant$ const $l_{n-1}$ и

$$
\varphi_{i}(z)= \begin{cases}\frac{z}{c_{i}^{2}\left(1-d_{i}\right)+d_{i}}, & z \in\left[0, d_{i}\right], \\ \frac{d_{i}\left(1-c_{i}^{2}\right)+c_{i}^{2} z}{c_{i}^{2}\left(1-d_{i}\right)+d_{i}}, & z \in\left[d_{i}, 1\right] .\end{cases}
$$

Подставляя (3.9) и (3.10) в (3.7) и используя условие $c_{1}^{2} c_{2}^{2} \ldots c_{m}^{2}=1$, получаем утверждение леммы 3.2. Лемма 3.2 доказана. 
ДОКАЗАТЕЛЬСТВО ТЕОРЕМЫ 3.1. Подставляя (3.4)-(3.6) в (3.3), получаем

$$
z_{q_{n}}\left(z_{0}\right)=\frac{M_{n} z_{0}}{1+\left(M_{n}-1\right) z_{0}}+\chi_{4}\left(z_{0}\right)
$$

где $\left\|\chi_{4}\left(z_{0}\right)\right\|_{1} \leqslant$ const $l_{n-1}^{\varepsilon}, M_{n}=M\left(q_{n}\right)$.

Легко проверить, что $z_{0}=-z$ и $f_{n}(z)=a_{n}-\left(a_{n}+b_{n}\right) z_{q_{n}}(-z)$, где

$$
z=\frac{y-y_{0}}{y_{0}-y_{q_{n-1}}}, \quad b_{n}=\frac{y_{0}-y_{q_{n-1}+q_{n}}}{y_{0}-y_{q_{n-1}}} .
$$

Отсюда и из (3.11) следует, что

$$
f_{n}(z)=\frac{a_{n}+\left(a_{n}+b_{n} M_{n}\right) z}{1+\left(1-M_{n}\right) z}+\chi_{5}(z)
$$

где $\left\|\chi_{5}(z)\right\|_{C^{1}([-1 ; 0])} \leqslant$ const $l_{n-1}^{\varepsilon}$.

Рассуждая так же, как и при выводе (3.12), можно показать (см. [8]), что

$$
g_{n}(z)=\frac{-a_{n}+\left(1-\bar{M}_{n} b_{n}\right) z}{a_{n}+\left(\bar{M}_{n}^{-1}-1\right) z}+\chi_{6}(z),
$$

где $\left\|\chi_{6}(z)\right\|_{C^{1}\left(\left[0 ; a_{n}\right]\right)} \leqslant$ const $l_{n}^{\varepsilon}, \bar{M}_{n}=\bar{M}\left(q_{n-1}\right)$. Используя тот факт, что

$$
\begin{aligned}
M_{n} \bar{M}_{n} & =\exp \left\{\sum_{i=0}^{q_{n}-1} \int_{y_{i+q_{n-1}}}^{y_{i}} \frac{f^{\prime \prime}(t)}{2 f^{\prime}(t)} d t+\sum_{j=0}^{q_{n-1}-1} \int_{y_{j}}^{y_{j+q_{n}}} \frac{f^{\prime \prime}(t)}{2 f^{\prime}(t)} d t\right\}= \\
& =\exp \left\{\int_{S^{1}} \frac{f^{\prime \prime}(t)}{2 f^{\prime}(t)} d t\right\}=\exp \left\{\frac{1}{2} \ln \left(c_{0}^{2} c_{1}^{2} \cdots c_{m}^{2}\right)\right\}=1,
\end{aligned}
$$

т.е. что $\bar{M}_{n}=M_{n}^{-1}$, окончательно получаем

$$
g_{n}(z)=\frac{-a_{n}+\left(1-M_{n} b_{n}\right) z}{a_{n}+\left(M_{n}-1\right) z}+\chi_{6}(z) .
$$

ЗАМЕчАНИЕ. При нечетном $n$ по-прежнему справедливы формулы (3.12) и (3.13), однако в этом случае

$$
M_{n}=\exp \left\{-\sum_{s=0}^{q_{n}-1} \int_{x_{s}}^{x_{s+q_{n-1}}} \frac{f^{\prime \prime}(t)}{2 f^{\prime}(t)} d t\right\}
$$

Для завершения доказательства теоремы 3.1 нам достаточно показать, что $M_{n}$ и $\left(a_{n}+b_{n}\right)$ стремятся к единице с экспоненциальной скоростью.

ЛЕмма 3.3. При $n>l$ справедливы неравенства

$$
\left|1-M_{n}\right| \leqslant \operatorname{const} \lambda^{n \varepsilon}, \quad\left|1-\left(a_{n}+b_{n}\right)\right| \leqslant \operatorname{const} \lambda^{n \varepsilon} .
$$


ДоКАЗАТЕЛЬСТво ЛЕмМЫ 3.3. Функции $f_{n}(z)$ и $g_{n+1}(z)$ отвечают одному и тому же преобразованию, но в разных координатах, т.е.

$$
g_{n}(z)=-\frac{1}{a_{n}} f_{n}\left(-a_{n} z\right) .
$$

Введем обозначения $c_{n}=1-M_{n}$ и $d_{n}=1-a_{n}-b_{n}$.

Теперь мы воспользуемся следуюшими рекуррентными формулами для $c_{n}$ и $d_{n}$, которые были получены в [8] при помощи (3.14):

$$
\begin{aligned}
c_{n}=a_{n} a_{n+1} c_{n-1}+\xi_{n}, & n>l\left(y_{0}\right), \\
d_{n}=-a_{n} d_{n-1}+a_{n} \bar{\xi}_{n}, & n>l\left(y_{0}\right),
\end{aligned}
$$

где $\left|\xi_{n}\right| \leqslant \operatorname{const} l_{n-1}^{\varepsilon}$ и $\left|\bar{\xi}_{n}\right| \leqslant \operatorname{const} l_{n-1}^{\varepsilon}$.

Итерируя (3.15), получим

$$
c_{n}=\sum_{i=l}^{n} \xi_{i} \prod_{j=i}^{n} \frac{a_{j}^{2}}{a_{n} a_{i}}=\sum_{i=1}^{n} \xi_{i} \frac{\left|\Delta_{0}^{(n)}\left(y_{0}\right)\right|\left|\Delta_{0}^{(n-1)}\left(y_{0}\right)\right|}{\left|\Delta_{0}^{(i)}\left(y_{0}\right)\right|\left|\Delta_{0}^{(i-1)}\left(y_{0}\right)\right|} .
$$

В силу леммы 2.1 имеем

$$
\frac{\left|\Delta_{0}^{(n)}\left(y_{0}\right)\right|\left|\Delta_{0}^{(n-1)}\left(y_{0}\right)\right|}{\left|\Delta_{0}^{(i)}\left(y_{0}\right)\right|\left|\Delta_{0}^{(i-1)}\left(y_{0}\right)\right|} \leqslant \lambda^{2(n-i)}
$$

Отсюда, используя неравенство $l_{n-1} \leqslant$ const $\lambda^{n}$, получаем

$$
c_{n} \leqslant \text { const } \sum_{i=l}^{n} \lambda^{i \varepsilon} \lambda^{2(n-i)} \leqslant \operatorname{const} \lambda^{n \varepsilon} \sum_{i=l}^{n} \lambda^{(2-\varepsilon)(n-i)} \leqslant \operatorname{const} \lambda^{n \varepsilon} \text {. }
$$

Тем самым первое утверждение леммы 3.3 доказано.

Неравенства $a_{n}+b_{n} \leqslant$ const, $\left|\bar{\xi}_{n}\right| \leqslant$ const $\lambda^{n \varepsilon}$ и итерирование (3.16) дают

$$
d_{n}=\sum_{i=l}^{n}(-1)^{(n-i)} \bar{\xi}_{i} \prod_{j=i}^{n} a_{j}=(-1)^{n} \sum_{i=l}^{n}(-1)^{i} \bar{\xi}_{i} \frac{\left|\Delta_{0}^{(n)}\left(y_{0}\right)\right|}{\left|\Delta_{0}^{(i-1)}\left(y_{0}\right)\right|},
$$

откуда следует второе утверждение леммы 3.3. Теорема 3.1 полностью доказана.

СлеДСтвИЕ. Пусть $y_{0} \in V \backslash\left\{x_{0}\right\}$ u $y_{k}=T_{f}^{k} y_{0}, k \geqslant 1$. Тогда для любого $n>l$ имеем

$$
e^{-\alpha_{n}} \leqslant \prod_{i=0}^{q_{n}-1} f^{\prime}\left(y_{i}\right) \leqslant e^{\alpha_{n}}
$$

əде $\alpha_{n}=$ const $\lambda^{n \varepsilon}$. 
ЗАмЕЧАниЕ. Если взять произвольную точку $y_{0} \in S^{1} \backslash\left\{x_{i}, i=\overline{0, p_{m}}\right\}$ и определить число $l=l\left(y_{0}\right)$ так, чтобы отрезок $\left[x_{q_{l-1}}, x_{q_{l}}\right]$ и его первые $p_{m}$ образов не содержали особых точкек, то справедливы теорема 3.1 и неравенство (3.17).

ДокАЗАТЕЛЬСТво СЛЕДСтвия. Дифференцируя (3.1), получаем

$$
\frac{d}{d x}\left(T^{q_{n}}\right)\left(x_{0}\right)=\prod_{i=0}^{q_{n}-1} f^{\prime}\left(y_{i}\right)=f_{n}^{\prime}(0) .
$$

В силу теоремы 3.1

$$
\left|f_{n}^{\prime}(0)-1\right| \leqslant \operatorname{const} \lambda^{n \varepsilon},
$$

откуда получаем требуемые неравенства.

\section{4. ДОКАЗАТЕЛЬСТВО ТЕОРЕМЫ 1.1}

При доказательстве теоремы 1.1 используются рассуждения, аналогичные тем, которые использовались при доказательстве основной теоремы в работе Синая и Ханина [8]. Далее (см. теорему 4.1 ниже) будет показано, что плотность $h(x)$ абсолютно непрерывной инвариантной меры является строго положительной функцией, непрерывной всюду, за исключением нескольких точек разрыва первого рода.

Используя эту информацию, а также неравенство (3.17), путем стандартных рассуждений мы можем легко доказать теорему 1.1 (см. [8]). Рассмотрим функциональное уравнение

$$
h\left(T_{f} x\right)=\frac{1}{f^{\prime}(x)} h(x) .
$$

Напомним, что решение (4.1) является плотностью инвариантной меры гомеоморфизма $T_{f}$.

Теорема 4.1. При выполнении условий теоремы 1.1 функииональное уравнение (4.1) имеет строго положительное, кусочно-непрерывное решение $h(x)$ такое, что:

1) $h(x) \in C\left(S^{1} \backslash\left\{x_{i}, 1 \leqslant i \leqslant p_{m}\right\}\right)$;

2) в точках $x=x_{i}, \quad 1 \leqslant i \leqslant p_{m}$, функиия $h(x)$ имеет разрыв первого рода:

$$
\frac{h\left(x_{i}-0\right)}{h\left(x_{i}+0\right)}=c_{j} c_{j+1} \ldots c_{m} \quad n p u \quad p_{j-1}<i \leqslant p_{j}, \quad j=\overline{1, m}
$$

Доказательство теоремы 4.1. Решение уравнения (4.1) на множестве $B=$ $\left\{x_{i}, i \geqslant p_{m}+1\right\}$ построим следующим образом:

$$
h\left(x_{p_{m}+1}\right)=1, \quad h\left(x_{i}\right)=\prod_{j=p_{m}+1}^{i-1}\left(f^{\prime}\left(x_{j}\right)\right)^{-1}, \quad i>p_{m}+1 .
$$


Поскольку множество $B$ всюду плотно на окружности, необходимо убедиться, что функцию $h(x)$ можно продолжить до непрерывной положительной функции на множестве $S^{1} \backslash\left\{x_{i}, 1 \leqslant i \leqslant p_{m}\right\}$. Мы сначала построим решение (4.1) в окрестности $V=\left[y_{q_{n_{0}-1}}, y_{q_{n_{0}}}\right]$ начальной особой точки $x_{0}$. Возьмем произвольную точку $y_{0} \in V$ и отрезок $\Delta_{0}^{(n-1)}\left(x_{i}\right), \quad i \geqslant p_{m}+1, \quad x_{i} \in V, \quad n \geqslant l+1$, содержаший точку $y_{0}$. Отрезок $\Delta_{0}^{(n-1)}\left(x_{i}\right)$ содержит внутри себя $k_{n+1}$ точек $x_{i+q_{n-1}+j q_{n}}, \quad 1 \leqslant j \leqslant k_{n+1}$, являюшихся концами элементов разбиения $\xi_{n+1}\left(x_{i}\right)$. Используя неравенство $(3.17)$, получаем

$$
\begin{aligned}
e^{-\alpha_{n}} & \leqslant \frac{h\left(x_{i+q_{n-1}+(j-1) q_{n}}\right)}{h\left(x_{i+q_{n-1}+j q_{n}}\right)}= \\
& =\prod_{s=i+q_{n-1}+(j-1) q_{n}}^{i+q_{n-1}+j q_{n}-1} f^{\prime}\left(x_{s}\right) \leqslant e^{\alpha_{n}}, \quad 1 \leqslant j \leqslant k_{n+1}, \\
e^{-\alpha_{n+1}} & \leqslant \frac{h\left(x_{i+q_{n-1}+k_{n+1} q_{n}}\right)}{h\left(x_{i}\right)}=\frac{h\left(x_{i+q_{n+1}}\right)}{h\left(x_{i}\right)} \leqslant e^{\alpha_{n+1}} .
\end{aligned}
$$

Отсюда следует, что для любой точки $x_{s} \in \Delta_{0}^{(n-1)}\left(x_{i}\right)$ имеем

$$
\exp \left\{-\sum_{m=n}^{\infty} k_{m+1} \alpha_{m}\right\} \leqslant \frac{h\left(x_{s}\right)}{h\left(x_{i}\right)} \leqslant \exp \left\{\sum_{m=n}^{\infty} k_{m+1} \alpha_{m}\right\} .
$$

Пусть $y_{0} \in\left[x_{s}, x_{s^{\prime}}\right] \subset \Delta_{0}^{(n-1)}\left(x_{i}\right)$, тогда

$$
\exp \left\{-2 \sum_{m=n}^{\infty} k_{m+1} \alpha_{m}\right\} \leqslant \frac{h\left(x_{s}\right)}{h\left(x_{s^{\prime}}\right)} \leqslant \exp \left\{2 \sum_{m=n}^{\infty} k_{m+1} \alpha_{m}\right\} .
$$

Пусть $k_{n} \leqslant$ const $n^{\alpha}, \alpha>0$. Поскольку $\alpha_{n}=$ const $\lambda^{n \varepsilon}$, ряд $\sum_{m=n}^{\infty} k_{m+1} \alpha_{m}$ сходится. Отсюда, а также из (4.5), следует, что для любой последовательности $\left\{x_{s_{n}}\right\} \subset$ $\Delta_{0}^{(n-1)}\left(x_{i}\right)$, сходящейся к $y_{0}$, верно, что

$$
\lim _{n \rightarrow \infty} h\left(x_{s_{n}}\right)=h\left(y_{0}\right),
$$

и, более того, для любой точки $x_{s_{n}} \in \Delta_{0}^{(n-1)}\left(x_{i}\right)$

$$
\exp \left\{-2 \sum_{m=n}^{\infty} k_{m+1} \alpha_{m}\right\} \leqslant \frac{h\left(y_{0}\right)}{h\left(x_{s}\right)} \leqslant \exp \left\{2 \sum_{m=n}^{\infty} k_{m+1} \alpha_{m}\right\}
$$

Рассмотрим разбиение $\xi_{n_{0}}\left(x_{0}\right)$. Мы показали, что на нулевых отрезках $\Delta_{0}^{\left(n_{0}-1\right)}\left(x_{0}\right)$ и $\Delta_{0}^{\left(n_{0}\right)}\left(x_{0}\right)$ функцию $h(x)$ можно продолжить по непрерывности. Отсюда, а также из (4.2) следует, что функцию $h(x)$ можно продолжить до непрерывной функции на всех отрезках разбиения $\xi_{n_{0}}\left(x_{0}\right)$ и в соответствующих точках верны оценки (4.6). Первое утверждение теоремы 4.1 доказано. Теперь покажем, что в точках $x=x_{i}, 1 \leqslant i \leqslant p_{m}$, 
функция $h(x)$ имеет разрыв первого рода. Фиксируем $x_{i}, p_{j-1}<i \leqslant p_{j}, 1 \leqslant j \leqslant p_{m}$. Возьмем $y_{1} \in\left[x_{i+q_{n-1}}, x_{i}\right)$ и $y_{2} \in\left(x_{i}, x_{i+q_{n}}\right], n \geqslant l$. Ясно, что

$$
\begin{aligned}
\frac{h\left(y_{1}\right)}{h\left(y_{2}\right)} & =\frac{h\left(y_{1}\right)}{h\left(x_{i+q_{n-1}}\right)} \frac{h\left(x_{i+q_{n}}\right)}{h\left(y_{2}\right)} \frac{h\left(x_{i+q_{n-1}}\right)}{h\left(x_{i+q_{n}}\right)}= \\
& =\frac{h\left(y_{1}\right)}{h\left(x_{i+q_{n-1}}\right)} \frac{h\left(x_{i+q_{n}}\right)}{h\left(y_{2}\right)} \frac{h\left(x_{q_{n-1}}\right)}{h\left(x_{q_{n}}\right)} \prod_{k=0}^{i-1} \frac{f^{\prime}\left(x_{k+q_{n}}\right)}{f^{\prime}\left(x_{k+q_{n-1}}\right)} .
\end{aligned}
$$

Используя условие "в" (см. введение) и оценку $\left|\Delta^{(n)}\right| \leqslant \operatorname{const} \lambda^{n}$, получаем

$$
\prod_{k=0}^{i-1} \frac{f^{\prime}\left(x_{k+q_{n}}\right)}{f^{\prime}\left(x_{k+q_{n-1}}\right)}=\prod_{k=0}^{i-1} c_{k}^{-1}\left[1+O\left(\lambda^{n}\right)\right] .
$$

Из (4.6)-(4.8), а также из условия “д” следует, что

$$
\frac{h\left(x_{i}-0\right)}{h\left(x_{i}+0\right)}=\prod_{s=j}^{m} c_{s}
$$

если $p_{j-1}<i \leqslant p_{j}, j=\overline{1, m}$, и

$$
\frac{h\left(x_{i}-0\right)}{h\left(x_{i}+0\right)}=1
$$

если $i \geqslant p_{m}+1$.

Теорема 4.1 доказана.

Благодарности. Автор выражает свою глубокую благодарность Я.Г. Синаю и К. М. Ханину за полезные обсуждения.

\section{Список литературы}

[1] И. П. Корнфельд, Я. Г. Синай, С. В. Фомин. Эргодическая теория. М.: Наука, 1980.

[2] A. Denjoy. J. Math. Pure Appl. 1932. V. 11. P. 333.

[3] В. И. Арнольд. Изв. АН СССР. Сер. матем. 1961. Т. 25. № 1. С. 21.

[4] M. Herman. Publ. Math. IHES. 1979. V. 49. P. 5.

[5] J. Moser. Ann. Scuole Norm. Sup. Pisa. Ser. III. 1966. V. 20. P. 409.

[6] Y. Katznelson, D. Ornstein. Ergodic theory Dyn. Systems. 1989. V. 9. № 4. P. 691.

[7] K. M. Khanin, Ya. G. Sinai. Commun. Math. Phys. 1987. V. 12. P. 89.

[8] Я.Г. Синай, К. М. Ханин. УМН. 1989. Т. 44. Вып.1. С. 57.

[9] J. Stark. Nonlinearity. 1988. V. 1. P. 541.

[10] Е. Б. Вул, К. М. Ханин. УМН. 1990. Т. 45. Вып. 3. С. 189.

[11] K. M. Khanin, E. B. Vul. Adv. Sov. Math. 1991. V. 3. P. 57.

[12] А. А. Джалилов, К. М. Ханин. УМН. 1996. Т. 51. № 6. С. 201.

[13] Я.Г. Синай. Современные проблемы эргодической теории. М.: Изд. фирма "Физ.-матем. литература", 1995. 